\title{
Patients with an ICD Can Safely Resume Work in Industrial Facilities Following Simple Screening for Electromagnetic Interference
}

\author{
OSNAT GUREVITZ, * RICHARD I. FOGEL, * MARK E. HERNER,† \\ ROSS SAMPLE,† ADAM S. STRICKBERGER,‡ EMILE G. DAOUD,‡ FRED MORADY,‡ \\ and ERIC N. PRYSTOWSKY* \\ From the *Indiana Heart Institute, Indianapolis, Indiana, +Guidant/CPI, St. Paul, Minnesota, \\ and $\neq$ University of Michigan, Ann Arbor, Michigan
}

GUREVITZ, O., ET AL.: Patients with an ICD Can Safely Resume Work in Industrial Facilities Following Simple Screening for Electromagnetic Interference. Patients with ICDs are commonly advised to quit industrial jobs because of concerns that strong electromagnetic fields operating in the industrial environment might interfere with ICD functions. This study was done to assess interactions between industrial equipment and ICDs, and to devise a simple low risk screening protocol. We studied 18 patients carrying nine different ICD models who were met at their workplace by a clinical technician and were asked to walk through their workplace and perform typical duties while sensing status was monitored by listening to the ICD's beeper. All devices were interrogated at the completion of testing. At follow-up, patients were contacted by phone and were asked about employment status and history of ICD discharges or syncope. One hundred eighty-four contacts with 114 types of industrial equipment in 13 different industrial facilities (including 31 contacts with arc welding machines) were monitored. Interference with ICD's function occurred in only one contact (0.5\%), when ICD therapy was temporarily suspended while a worker was attaching a huge electromagnet to a crane. At follow-up $46.0 \pm 6.0$ months after testing, 7 patients $(41 \%)$ are still holding the same job, 7 have retired because of reasons unrelated to their ICD, and 3 patients were transferred to a nonindustrial job. None of the patients had either an ICD shock or syncope during work. The use of a simple screening procedure can safely identify sources of electromagnetic interference that may affect ICD operation, and can predict long-term safety of working in an industrial workplace for ICD patients. (PACE 2003; 26:1675-1678)

\section{implantable cardioverter defibrillator, electromagnetic interference, oversensing}

\section{Introduction}

Implantable cardioverter defibrillators (ICDs) reduce mortality in patients with malignant ventricular arrhythmias. ${ }^{1-5}$ However, ICDs can have a negative impact on quality of life measures. ${ }^{6}$ One of the most important factors influencing the quality of life after a medical intervention is the ability to return to work. ${ }^{7}$ Patients with ICDs are often advised to quit industrial jobs because of concerns that strong electromagnetic fields operating in the industrial environment might interfere with the device's normal function.

Electromagnetic interference (EMI) can alter ICD's function in several ways: (1) Electrical signals within the frequency range of the ICD's sensing circuitry can induce false detection of arrhythmias and result in inappropriate therapy. ${ }^{8,9}$ (2) Sensed external electromagnetic noise may prevent detection of actual ventricular arrhythmias,

Address for reprints: Osnat Gurevitz, M.D., Heart Institute, Sheba Medical Center, Tel-Hashomer 52621, Israel. Fax: 9723-5356605; e-mail: ossigur@hotmail.com

Received July 15, 2002; revised September 4, 2002; accepted September 12, 2002. preventing the delivery of lifesaving therapy. ${ }^{10}$ (3) EMI can cause the defibrillator to revert to a "protection mode" changing its programmed parameters to the default values. ${ }^{10}$ (4) A strong magnetic field can close the reed switch of the device, causing it to be inhibited. ${ }^{11}$ The purpose of our investigation was to devise a simple, low risk screening protocol that will help ICD patients, physicians, and employers assess the risks of EMI in the industrial workplace.

Methods
Patients and Devices
The study group was comprised of 18 pa-
tients with ICDs ( 3 women) with a mean age of
$51.4 \pm 14.7$ (range: $23-77$ ) years. All patients re-
ceived an ICD because of documented sustained
ventricular arrhythmias, and none had a pacing
indication. Nine different models of ICD's were
implanted (Table I). All ICDs were manufactured
by Guidant/CPI (St. Paul, MN, USA). The devices
used can be programmed to emit an audible tone
for each sensed QRS and a special tone if EMI is
sensed. This feature enables continuous monitor-
ing of the device's sensing during daily activities




\begin{tabular}{lc}
\hline \multicolumn{2}{c}{ Table I. } \\
ICD Models* \\
\hline ICD model & \\
\hline Ventak 1555 & No. of patients \\
PRX 1705 & 1 \\
PRX 1720 & 1 \\
PRX 1725 & 3 \\
Mini 1740 & 1 \\
Mini 1743 & 2 \\
Mini 1762 & 3 \\
Mini 1763 & 3 \\
AV 1810 & 2 \\
\hline
\end{tabular}

*All devices were manufactured by Guidant/Cardiac Pacemaker, Inc., St. Paul, MN.

without the need to apply a magnet or suspend therapy. Fourteen patients had pectoral implants. All but one used a nonthoracotomy Endotak lead system (Guidant/CPI).

\section{Testing Procedure}

The study involved three stages:

Stage 1: At baseline all devices were interrogated. The magnet response was tested and the markers, magnet, and QRS beeper were enabled. One to one sensing correlating QRS detection and the beep tones were verified. Detection duration was extended to 15 seconds for each therapy zone while the ICD remained fully active. We did not feel that it was safe to inactivate ICD therapy without an external defibrillator immediately available, and thus chose to extend the arrhythmia detection duration for each therapy zone.

Stage 2: Each patient was asked to walk through their workplace and operate each item of equipment as they would during their usual activities. The device's QRS beeper was monitored with a stethoscope by a clinical engineer or nurse specially trained to work with ICDs. Each item was recorded as well as the approximate distance to the patient and the effects on the device's sensing. The engineer or nurse was instructed to apply a magnet and remove the patient from a source should oversensing occur.

Stage 3: At the conclusion of testing, all programmed parameters were returned to their initial values. The devices were interrogated and final parameters were compared with the initial values.

\section{Follow-Up}

Seventeen patients were contacted by phone $46 \pm 6$ months after initial testing. One patient was lost to follow-up. Patients were asked to give details regarding current employment status, reasons for retirement, and history of ICD shocks and syncopal spells during work. If a patient reported being shocked or experiencing syncope during work, the relevant pacemaker clinic was contacted and data regarding the event obtained.

\section{Results}

\section{Testing Results}

Between October 1995 and February 1998, 18 patients with ICDs requested testing in order to resume 11 different job positions at 13 different industrial facilities (Table II). In these patients, 184 contacts with 114 different types of equipment were recorded, including 31 with arc welding machines. The distance measured between the patient and the equipment ranged from zero (touching in 5 patients) to 360 inches, with a mean of $24.8 \pm 54.8$ inches. In only one instance was interference recorded when a worker in a steel facility was responsible for attaching a huge electromagnet to a crane. In this patient ICD therapy was temporarily inhibited while within 6 feet of the electromagnet. He was advised to change jobs.

Table II.

Job Positions and Industrial Facilities

\begin{tabular}{cll}
$\begin{array}{l}\text { Patient's } \\
\text { number }\end{array}$ & \multicolumn{1}{c}{ Job position } & \multicolumn{1}{c}{ Facility type } \\
\hline 1 & Engineer & $\begin{array}{l}\text { Electrical automobile } \\
\text { factory } \\
\text { Auto assembly }\end{array}$ \\
2 & Jobsetter & College \\
3 & Welder & Automobile steel factory \\
4 & Production worker & Metal factory \\
5 & Machinist & Electrical company \\
6 & Data processing & Metal factory \\
7 & Electrician & Machinery shop \\
8 & Production worker & Welding \\
9 & Production worker & Metal factory \\
10 & Production worker & Steel factory \\
11 & Production worker & Ster \\
12 & Welder & Machinery shop \\
13 & Press operator & Metal factory \\
14 & Electrician & Hospital \\
15 & Inspector & University \\
16 & Hobby & Home \\
17 & Radio operator & Home \\
18 & Machinist & Machining plant \\
\hline & &
\end{tabular}




\begin{tabular}{lcclc}
\hline \multicolumn{5}{c}{$\begin{array}{c}\text { Table III. } \\
\text { Follow-Up }\end{array}$} \\
\hline $\begin{array}{l}\text { Patient's } \\
\text { number }\end{array}$ & $\begin{array}{c}\text { Age (years) } \\
\text { sex }\end{array}$ & $\begin{array}{c}\text { Length of follow-up } \\
\text { (months) }\end{array}$ & Current occupation & $\begin{array}{c}\text { Shocks/syncope } \\
\text { at work }\end{array}$ \\
\hline 1 & $63 / \mathrm{M}$ & 60 & Retired & No \\
2 & $45 / \mathrm{F}$ & 59 & Non-industrial & No \\
3 & $29 / \mathrm{M}$ & 58 & Non-industrial & No \\
4 & $45 / \mathrm{M}$ & 57 & Industrial & No \\
5 & $76 / \mathrm{M}$ & & Lost to follow-up & No \\
6 & $23 / \mathrm{F}$ & 51 & Retired & No \\
7 & $45 / \mathrm{M}$ & 49 & Industrial & No \\
8 & $56 / \mathrm{M}$ & 48 & Industrial & No \\
9 & $33 / \mathrm{M}$ & 49 & Non-industrial & No \\
10 & $64 / \mathrm{M}$ & 47 & Industrial & No \\
11 & $49 / \mathrm{M}$ & 44 & Retired & Industrial \\
12 & $45 / \mathrm{M}$ & 42 & Retired & No \\
13 & $62 / \mathrm{F}$ & 39 & Retired & No \\
14 & $59 / \mathrm{M}$ & 38 & Retired & No \\
15 & $52 / \mathrm{M}$ & 38 & Industrial & No \\
16 & $59 / \mathrm{M}$ & 38 & Industrial & \\
17 & $77 / \mathrm{M}$ & 33 & & \\
18 & $44 / \mathrm{M}$ & 32 & & \\
Mean \pm SD & $51.4 \pm 14.7$ & $46.0 \pm 9.0$ & & \\
\hline
\end{tabular}

${ }^{*}$ Age at the time of testing. SD = standard deviation.

There were no other cases of oversensing in the other 183 contacts that were monitored. Similarly, we did not observe any changes in the devices' programmed parameters after any of these encounters.

\section{Follow-Up}

Follow-up data were obtained for $17(94 \%)$ patients $46.0 \pm 9.0$ (range $32-60$ ) months after testing (Table III). One patient was lost to followup. At the time of follow-up, 7 (41\%) patients were still working in the same position they held at the time of testing. Seven patients had retired $15.9 \pm 10.0$ months after testing. Reasons for retirement included: congestive heart failure in two patients, age, inner ear disease, cerebrovascular event, asthma, and childbearing in one patient each. Three patients changed to a nonindustrial job 5,23 , and 25 months after testing. Two of them said that they preferred to avoid the industrial workplace because they were concerned about potential interactions with their devices. None of them reported being shocked or experienced loss of consciousness while at work. During the follow-up period, only one patient reported having an ICD discharge while at work. Data obtained from his pacemaker clinic revealed no shock episodes according to the device's interrogation, and reproduction of the same "shock" sensation occurred by bending the head. The symptoms were attributed to vertebral discopathy. This patient continued to work as a welder at the time of follow-up 37 months after the reported "event." No patient reported syncope during work.

\section{Discussion}

\section{Electromagnetic Interference in the Workplace}

Interference of environmental electromagnetic fields with the function of cardiac pacemakers is well reported. ${ }^{12}$ Since ICDs became widely used in the mid 1980s, several case reports have described either the inappropriate delivery of shocks ${ }^{8,9}$ or the inactivation of devices by EMI. ${ }^{11}$ So far only few studies systematically addressed the effects of EMI in the workplace on patients with implanted devices. Marco et al. ${ }^{13}$ investigated 12 pacemaker patients in the industrial environment. They found that arc welding machines delivering up to 225 A did not adversely interfere with pacemaker function. However, a submerged arc welder using up to 1,000 A inhibited his pacemaker when the pacemaker was within 15 centimeters of the welding site. In a recent study, Fetter et al. ${ }^{14}$ interrogated ICDs of 11 patients immediately after they had operated different arc welding machines or were working near high power 
motors. In none of their cases did oversensing occur. Our findings are in agreement with these data. During routine work in industrial facilities, we were able to record oversensing by an ICD in only one of 184 encounters $(0.5 \%)$. We did not detect any reprogramming or reed switch closure. Differing from previous studies, we provide long-term follow-up. Our follow-up results help support the findings of initial testing and provide evidence for the reliability of our testing protocol. All but one patient were allowed to return to their previous work after testing. None of them had ICD discharges or syncope during work, and 46 months after testing, $41 \%$ of them still work in the industrial setting.

\section{Factors Influencing Interference}

The main factors determining whether an electromagnetic source will interfere with an ICD's functions are the frequency of the emitted electrical signals, the magnitude of the magnetic field, and the spatial orientation and proximity of the source to the device. Because one of our goals was to devise a simple and practical way of screening, we did not measure the electromagnetic fields operating in our patients' workplaces. However, Fetter et al. ${ }^{14}$ recorded the power rating of electrical equipment very similar to that used by our patients. According to their report, AC arc welders use from 2 to 375 A while DC arc welding machines use up to $900 \mathrm{~A}$. The magnetic flux density they measured at the surface of cables or motors ranged between 40 and $2,530 \mathrm{G}$ and decreased to 0.5 to $1.2 \mathrm{G}$ at a distance of 1 to 2 feet from the surface. The minimal magnet field that is required to cause reed switch closure (i.e, inactivation) of an

\section{References}

1. Moss AJ, Hall AJ, Cannon DS, et al, for the MADIT Investigators. Improved survival with an implanted defibrillator in patients with coronary disease at high risk for ventricular arrhythmia. N Engl J Med 1996; 335:1933-1940.

2. AVID Investigators. A comparison of antiarrhythmic drug therapy with implantable defibrillators in patients resuscitated from near-fatal arrhythmias. N Engl J Med 1997; 337:15761583

3. Buxton AE, Lee KL, Fisher JD, et al. A randomized study of the prevention of sudden death in patients with coronary artery disease: Multicenter Unsustained Tachycardia Trial Investigators. N Engl Med 1999; 341:1882-1890.

4. Connolly SJ, Gent M, Roberts RS, et al. Canadian Implantable Defibrillator Study (CIDS): A randomized trial of the implantable cardioverter defibrillator against amiodarone. Circulation 2000; 101:1297-1302.

5. Kuck KH, Cappato R, Siebels J, et al. Randomized comparison of antiarrhythmic drug therapy with implantable defibrillators in patients resuscitated from cardiac arrest. The Cardiac Arrest Study Hamburg (CASH). Circulation 2000; 102:748-754.

6. May CD, Smith PR, Murdock CJ, et al. The impact of the implantable cardioverter defibrillator on quality of life. PACE 1995; 18:14111418.

7. Kalbfleisch KR, Lehmann MH, Steinman RT, et al. Reemployment
ICD is $10 \mathrm{G}$. No oversensing occurred when ICD patients were instructed to keep a minimal distance of 1 foot from the equipment they were using. ${ }^{14}$ In contrast, we did not instruct our patients about the distance they should keep from their equipment, but instead recorded the actual distance they kept during usual daily tasks. With an average distance of $24.8 \pm 54.8$ inches (ranging 0-360 inches) no oversensing was recorded during 183 of 184 contacts monitored. ICD therapy was temporarily suspended at a distance of up to 6 feet from a very powerful electromagnet in one patient.

\section{Study Limitations}

One limitation of our study is that devices from only one manufacturer (CPI/Guidant) were assessed. It is possible that other ICDs and lead systems might respond differently. Also, electromagnetic fields in a specific workplace may change over time because of wear and tear or the replacement of machines. A change in the patient's distance from a machine or mode of operation can also influence the probability of EMI. Thus, alterations in the workplace may warrant a new testing procedure.

\section{Conclusions}

Our findings suggest that patients who receive an ICD, typically can resume work in industrial facilities after appropriate individual testing is performed. In the event that the patient experiences lightheadedness or a shock, they must be removed immediately from the source and seek medical evaluation. Any change made in the equipment operated by the patient or in their ICD warrants retesting for possible interactions.

following implantation of the automatic cardioverter defibrillator. Am J Cardiol 1989; 64:199-202.

8. Man KC, Davidson T, Langberg JJ, et al. Interference from a handheld radiofrequency remote control causing discharge of an implantable defibrillator. PACE 1993; 16:1756-1758.

9. Mathew P, Lewis C, Neglia J, et al. Interaction between electronic article surveillance systems and implantable defibrillators: Insights from a fourth generation ICD. PACE 1997; 20:2857-2859.

10. Barbaro V, Bartolini P, Bellocci F, et al. Electromagnetic interference of digital and analog cellular telephones on implantable cardioverter defibrillators: In vitro and in vivo studies. PACE 1999; 22:626-634.

11. Schmitt C, Brachmann J, Waldecker B, et al. Implantable cardioverter defibrillator: possible hazards of electromagnetic interference. PACE 1991; 14:982-984.

12. Carleton RA, Sessions RW, Graettinger JS. Environmental influence on implantable cardiac pacemakers. J Am Coll Med Assoc 1964; 190:938-940.

13. Marco D, Eisinger G, Hyes DL. Testing of work environments for electromagnetic interference. PACE 1992; 15: 2016-2022.

14. Fetter JG, Benditt DG, Stanton MS. Electromagnetic interference from welding and motors on implantable cardioverter-defibrillators as tested in the electrically hostile work site. J Am Coll Cardiol $1996 ; 28: 423-427$ 